\title{
Experimental Validation of a 3D Dynamic Finite-Element Model of a Total Knee Replacement
}

\author{
Joel L. Lanovaz ${ }^{1}$ and Randy E. Ellis ${ }^{1,2,3}$ \\ 1 Department of Mechanical and Materials Engineering, \\ Human Mobility Research Center, Queen's University, Canada \\ lanovaz@me.queensu.ca \\ 2 School of Computing, Queen's University, Canada \\ ellis@cs.queensu.ca \\ 3 Division of Orthopaedics, Department of Surgery, Queen's University, Canada
}

\begin{abstract}
A 3D forward-dynamics model of a total knee replacement was developed using an explicit finite-element package. The model incorporated both a tibiofemoral and a patellofemoral joint and allowed full 6-DOF kinematics for both joints. Simulated quadriceps contraction was used to drive the model. For validation, a unique experimental apparatus was constructed to simulate an openchain extension motion under quadriceps control. The ligamentous constraints of the MCL and LCL were simulated using tension springs. The kinematics of the tibia and patella were recorded along with the net forces and moments applied to the femur. Several ligament and inertial configurations were simulated. The RMS differences between the experimental data and model predictions across all simulations were excellent for both the kinematics (angles: $0.3-1.6^{\circ}$, displacements: $0.1-0.8 \mathrm{~mm}$ ) and kinetics (forces: $5-11 \mathrm{~N}$, moments: $0.2-0.6 \mathrm{Nm}$ ). The validated model will be extended with physiologically realistic ligaments and utilized in surgical planning simulations.
\end{abstract}

\section{Introduction}

Even though total knee replacement (TKR) is a relatively routine orthopedic procedure, it has been demonstrated that the introduction of image guided and computer assisted surgery (CAS) techniques to this task can decrease the variability of standard operations and increase the potential for good outcomes in complicated situations [1, 2].

Preoperative planning with current CAS systems utilize component manufacturer's guidelines, standard orthopedic practices and the surgeon's experience to select optimal component positioning. These criteria can be difficult to apply in complex procedures and may not fully take into account dynamic or loading effects that could accelerate component wear.

Intraoperative placement with CAS systems precisely reproduces the preoperative plan. However, the final in vivo performance of the components is still difficult to assess. Ligaments and soft tissue balancing can change the expected kinematics, leading to increased wear and decreased component life.

The focus of this research is to develop a realistic, functionally based dynamic TKR model that can assist in both the planning and execution of the surgery. Ultimately, the goal is to provide the surgeon with the ability to predict the postoperative behavior of TKR components based on preoperative and intraoperative data. 
Dynamic finite element (FE) analysis is becoming a popular modeling technique for examining various aspects of TKR mechanics [3, 4, 5, 6, 7]. Most current models focus on simulations for the purpose assessing wear behavior, usually by modeling a wear testing standard. All of the dynamic FE models presented in the literature to date utilize some prescribed kinematics as a control method. Few FE models incorporate both the tibiofemoral and patellofemoral joints [7].

This paper presents an explicit dynamic FE model of a TKR. The model incorporates the femoral, tibial and patellar components and is driven by simulated quadriceps action. A unique experimental validation procedure is described which simulates ligament constraints and assesses both the kinematic and kinetic performance of the model.

\section{Materials and Methods}

This study modeled posterior-cruciate-retaining TKR components (Sigma PFC, Depuy Orthopaedics, Indiana, U.S.A.). A standard right side, size 3 femoral component was used with a corresponding $10 \mathrm{~mm}$ thick tibial UHMWPE insert. A standard $10 \mathrm{~mm}$ thick UHMWPE patellar resurfacing button was also used.

\subsection{Experimental Apparatus}

An experimental apparatus was designed to simulate an open-chain knee extension motion (Fig. 1). The device incorporated a fully mobile patella and shank, with an actuatorcontrolled quadriceps and a simulated patellar tendon. The medial and lateral ligament constraints were also simulated.

The base of the apparatus consisted of a 6-DOF force transducer (MC3A-250, AMTI, Massachusetts, U.S.A.) mounted on a rigid aluminum frame. The femoral component was fixed to a mounting block which was secured to the free end of the force transducer such that the femoral long axis was parallel to the horizontal and the trochlear groove was facing up.

To remove the confounding effects of back-side motion, the tibial component was fixed directly to a mounting block which was bolted to the proximal end of a simulated shank segment. The shank segment consisted of 4 threaded rods, each $300 \mathrm{~mm}$ long, held in place by Plexiglas end plates. A $2.2 \mathrm{~kg}$ mass was fixed on the center line of the shank at the distal end.

Steel tension springs were used to simulate ligament constraints of the knee joint. Spherical rod-end bearings were bolted to Plexiglas plates that were rigidly attached to the frame on the medial and lateral sides of the femoral component. Bearings were also bolted to custom-fabricated ABS plastic mounting blocks fixed to the shank. Each tension spring was attached to a femur bearing on one end and a shank bearing on the other. In this way, ligament constraints similar to the MCL and LCL could be represented.

The springs were nearly identical and calibration showed a linear stiffness throughout the operating range (average $1.36 \mathrm{~N} / \mathrm{mm}$, SD $0.01 \mathrm{~N} / \mathrm{mm}$ ). Each spring produced tensile forces in the range of $40 \mathrm{~N}$ to $60 \mathrm{~N}$ during the various experimental trials.

The quadriceps and patellar tendon were simulated using $1.6 \mathrm{~mm}$ diameter steel cables. One end of the quadriceps cable was attached, via low friction pulleys, to the 


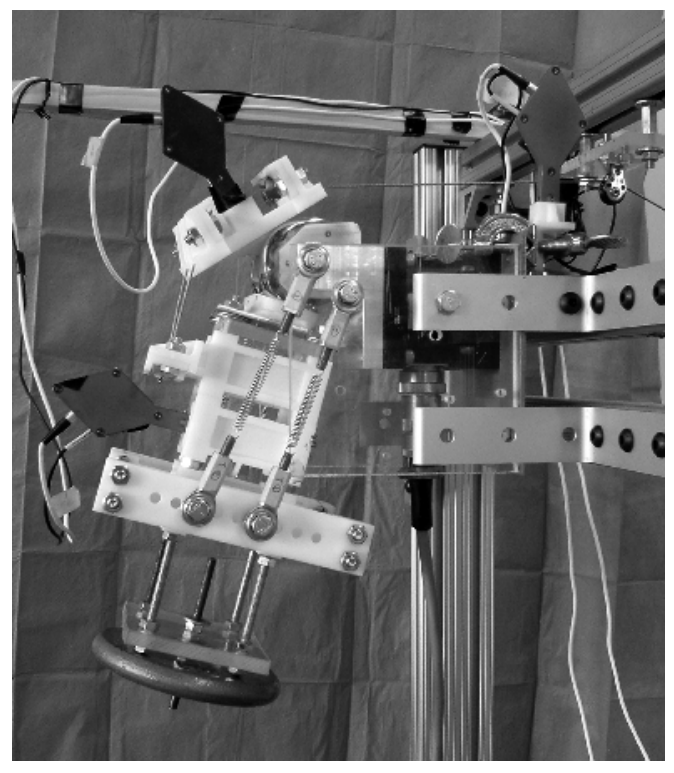

Fig. 1. Experimental apparatus in a 4 spring symmetrical configuration

cross head of a computer-controlled material testing machine (Model 5500R, Instron, Massachusetts, U.S.A.). Two $90 \mathrm{~mm}$ lengths of cable were used to simulate the patellar tendon.

A custom mounting block for the patellar button was fabricated from ABS plastic, which included attachment points for the quadriceps cable and the patella tendon cables. The cable attachments were lubricated ABS spherical bearings. Attachment points for the patellar tendon cables were also mounted on the shank segment. Once assembled, the motion of the joint complex was restricted so that the patella button remained in contact with the trochlear groove on the femoral component.

In order to validate the FE model, both kinematic and kinetic variables were experimentally measured. Optical tracking arrays were rigidly fixed to the shank, the patella mounting block and to the apparatus frame. An Optotrak 3020 system (NDI, Waterloo, Canada) was used to record the 3D kinematic data. The components were located relative to their respective tracking arrays through fiducial and shape matching registration based on points obtained with a calibrated optical tracking probe. The location and orientation of the force transducer, along with the line of action of the quadriceps cable, were also obtained with the probe. Insertion locations of the springs and cables were known in the coordinate frames of the respective rigid bodies to which they were attached.

For the dynamic experimental trials, the kinematic data and the conditioned voltage signals from the force transducer were collected simultaneously with the Optotrak system at a sampling rate of $100 \mathrm{~Hz}$.

For comparison with the FE model, all kinematic data were transformed into the transducer coordinate frame with the positive $\mathrm{X}$-axis corresponding to the femoral 
medial direction, the positive Y-axis to the femoral anterior direction and the positive $\mathrm{Z}$-axis to the femoral distal direction.

\subsection{Finite Element Model}

Three dimensional digital point cloud representations of the TKR component geometries were obtained by laser scanning (nominal scan resolution $0.05 \mathrm{~mm}$ ). Commercial software (Imageware, EDS Corp., Texas, U.S.A.) was used to fit surfaces (fit tolerance of $0.1 \mathrm{~mm}$ ) to the 6 contact regions ( 3 for the femur, 2 for the tibia and 1 for the patella) of each of the 3 components. The contact surfaces were saved in IGES format and transferred to meshing software (CUBIT, Sandia National Laboratories, New Mexico, U.S.A.).

Quadrilateral meshes were constructed for each contact surface. Pilot studies examining model convergence showed that a stable solution could be found for this model using a nominal element edge size of $1.0 \mathrm{~mm}$. This resulted in approximately 925 quadrilaterals representing each of the tibial contact regions, 1550 quadrilaterals representing each of the femoral contact regions and 950 quadrilaterals representing the patella. Hexahedral meshes for all components were generated from the quadrilateral meshes using custom routines written in Matlab (Mathworks, Massachusetts, U.S.A.).

The dynamic explicit FE package LS-DYNA (Livermore Software Technology Corp., California, U.S.A.) was utilized as the solver. A non-linear elastic-plastic deformable material model was used for all of the UHMWPE components [5, 6]. Because the elastic modulus of the CoCrMo femoral component was more than two orders of magnitude greater than the UHMWPE, the femoral component was modeled as a rigid material. The full thickness of the UHMWPE components were modeled, whereas the femoral components were made $1.5 \mathrm{~mm}$ thick (for visualization purposes only). A rigid layer of elements was fixed to the bottom layer of the UHMWPE elements in both the tibial and patella meshes to simulate the boundary conditions in the experimental apparatus.

The springs and cables were modeled with linear tension-only beam elements. The actuation of the quadriceps cable was modeled using a linear motor joint.

\subsection{Model Validation}

Four configurations were tested experimentally. The first had one medial and one lateral spring with symmetrical insertions. The second was a two-spring asymmetrical configuration with the shank insertions placed anterior to the femur insertion on the medial side and posterior to the femur insertion on the lateral side. The third configuration had the same spring insertions as the first but with an additional $0.5 \mathrm{~kg}$ mass fixed at the distal end of the shank, offset $150 \mathrm{~mm}$ to the medial side. The final configuration was a symmetrical four-spring design.

Average results were obtained from four trials for each configuration. For each trial, the knee joint started in the most flexed position with the patella button still in contact with the femur. The material testing machine was programmed to pull the quadriceps cable at a constant rate of $16 \mathrm{~mm} / \mathrm{s}$ for 2.5 seconds. This produced an extension of the 
knee joint from $60^{\circ}$ to $25^{\circ}$ of flexion over that time period. For all trials, the contact surfaces were lubricated with $20^{\circ} \mathrm{C}$ distilled water.

The FE model simulated the complete inertial properties of the shank and patella, including the tracking arrays. The solution was run in explicit mode with a time step of $10 \mu \mathrm{s}$. The initial poses of the component models were obtained from the experimental data. A $500 \mathrm{~ms}$ period was given at the start of each solution run to stabilize contact prior to initiating the cable actuator. A penalty-based contact algorithm was selected in LS-DYNA that calculated reaction forces based on nodal penetration and material characteristics. A dynamic coefficient of friction of 0.10 was applied to each of the three $\mathrm{CoCrMo/UHMWPE} \mathrm{contact} \mathrm{regions} \mathrm{in} \mathrm{the} \mathrm{model;} \mathrm{this} \mathrm{value} \mathrm{was} \mathrm{obtained} \mathrm{through} \mathrm{pilot}$ studies and is consistent with most values from the literature [3, 4, 5].

\section{Results}

The FE model was first evaluated by comparing the predicted 3D kinematics of the tibia (Fig. 2) and patella (Fig. 3) to the experimental values. The kinematic data were all calculated with respect to the force transducer coordinates. The rotations were expressed using three Cardan angles while the displacements were expressed relative to the transducer origin.

The nodal contact forces applied to the femur model were used to predict the net forces and moments recorded by the force transducer (Fig. 44).

The RMS differences between the experimental data and the FE predictions for all 4 configurations were calculated (Table 1). The maximum relative RMS differences occurred when predicting the rotational kinematics of the patella and these were mainly due to offsets that occurred during the initialization phase.
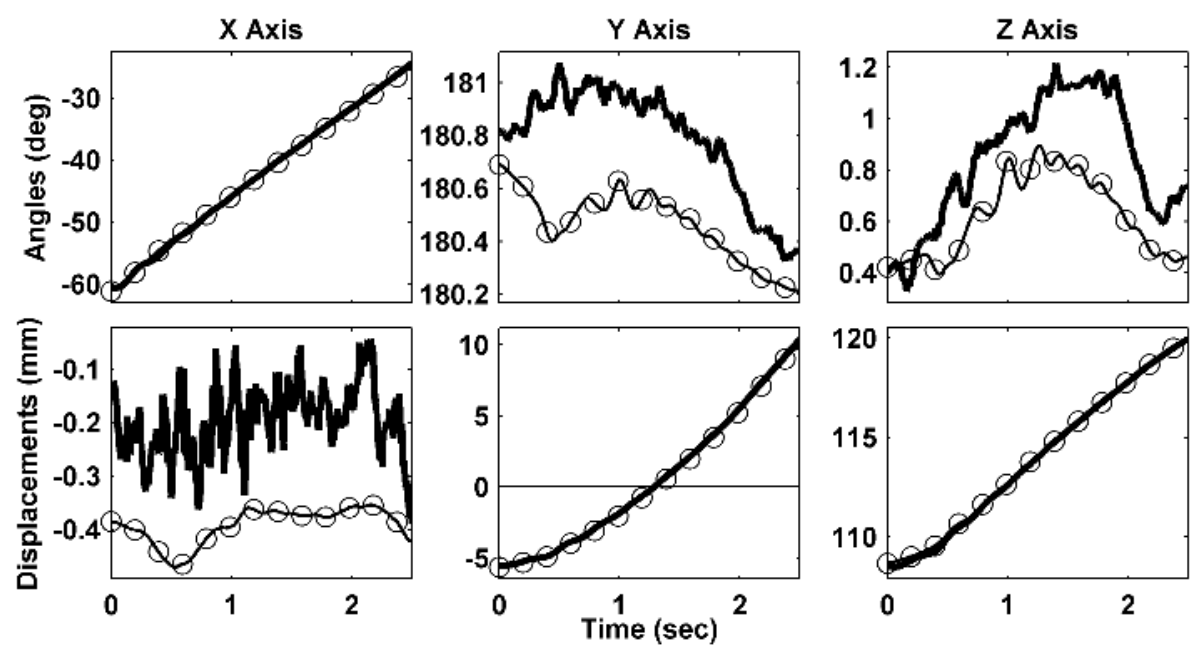

Fig. 2. Tibial kinematics comparing experimental (solid lines) and FE results (circles) from the two spring symmetrical configuration 

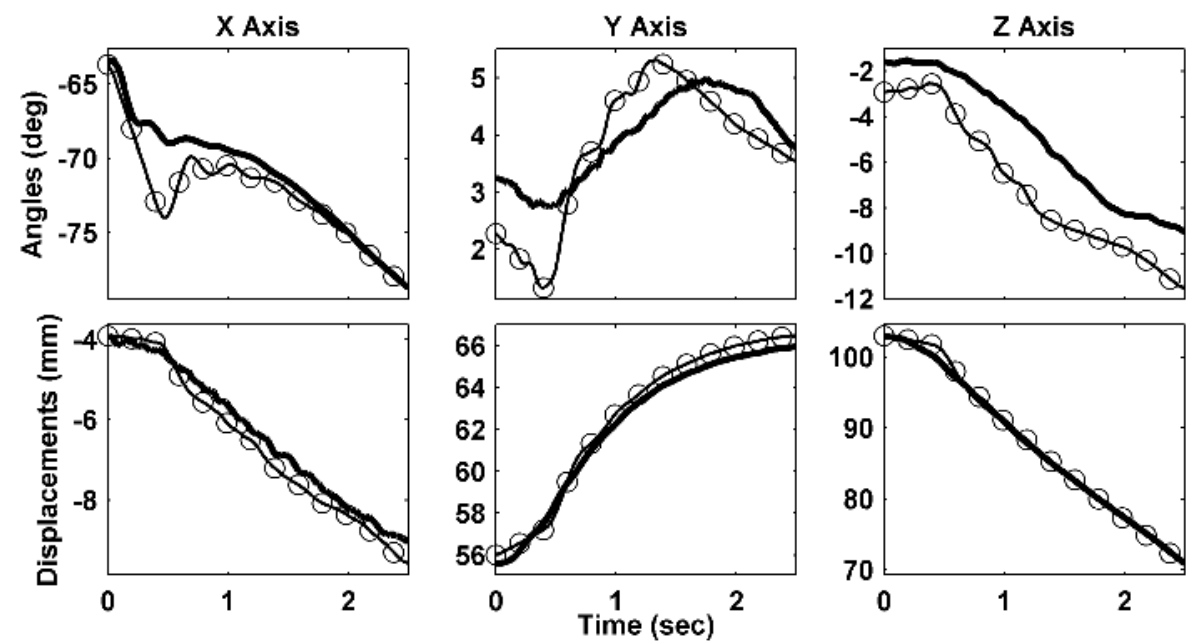

Fig. 3. Patellar kinematics comparing experimental (solid lines) and FE results (circles) from the two spring symmetrical configuration
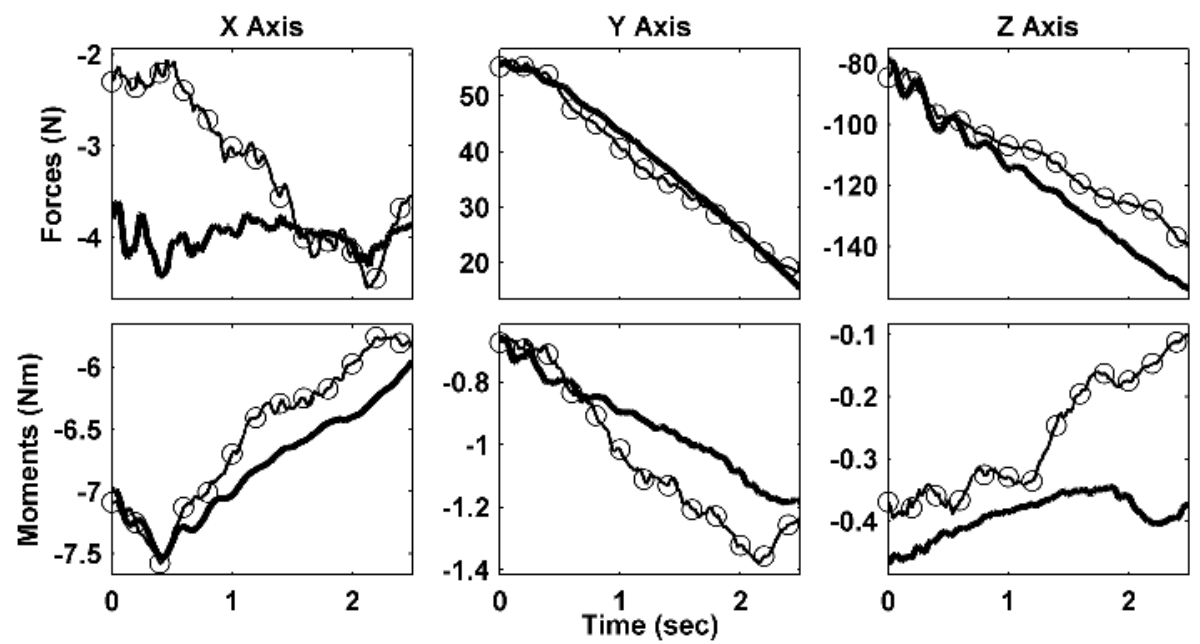

Fig. 4. Comparison between experimentally measured net forces and moments applied to the femur (solid lines) and the FE results (circles) from the two spring symmetrical configuration

\section{Discussion}

We wished to validate the FE model by simulating an open-chain knee extension motion under simulated quadriceps control. The difficulty in obtaining accurate geometric data and material properties during in vitro studies suggested that a more controlled mechanical simulation would be a better alternative. The experimental apparatus used 
Table 1. The RMS differences between the experimental data and FE models. The angle data are the means of the 3 axes. All other data are vector sums. Model 1: 2 symmetrical springs, Model 2: 2 asymmetrical springs, Model 3: 2 symmetrical springs with offset mass, Model 4: 4 symmetrical springs.

\begin{tabular}{|c|c|c|c|c|c|c|}
\hline \multirow{2}{*}{ Model } & \multicolumn{2}{|c|}{ Tibia } & \multicolumn{2}{|c|}{ Patella } & \multicolumn{2}{|c|}{ Femoral Transducer } \\
\hline & Angle (deg) & Position (mm) & Angle (deg) & ) Position (mm) & Force $(\mathrm{N})$ & Moment $(\mathrm{Nm})$ \\
\hline 1 & 0.31 & 0.16 & 1.58 & 0.50 & 10.04 & 0.29 \\
\hline 2 & 0.46 & 0.20 & 1.23 & 0.48 & 11.14 & 0.26 \\
\hline 3 & 0.47 & 0.27 & 1.05 & 0.41 & 5.04 & 0.34 \\
\hline 4 & 0.43 & 0.13 & 1.05 & 0.74 & 6.27 & 0.56 \\
\hline
\end{tabular}

in this study enabled the simulation of ligament-like constraints with the advantage of having precise material characteristics.

The FE model performed well under all 4 test conditions. The tibial kinematics were predicted with a high degree of fidelity. The RMS differences for the tibial displacements and angles is at the limit of the accuracy of the Optotrak system. The patellar angle predictions showed the highest RMS differences but were still under $2^{\circ}$ and closely followed the shape of the experimental data. It is not unreasonable to expect less accuracy predicting the patellar kinematics due to its low relative mass. Under the higher loading conditions of the 4 spring configuration, the patellar predictions improved.

Direct validation of dynamic contact models is difficult. Pressure measurement systems are available but can alter the contact characteristics [8]. One of the motivations in collecting the net forces and moments applied to the femur was to provide an indirect assessment of the contact data in the model. Because both the kinematics and kinetics were reproduced faithfully, we can assume that the contact information in the model is valid. This is a key component in this work because, in the future, the model will be used to calculate forward dynamics based on functional control inputs (such as muscle forces) as opposed to prescribed kinematic parameters (such as flexion angle).

LS-DYNA has the capability, similar to other FE packages [7], of switching to an all-rigid analysis without having to change the model. In this mode, kinematics and contact pressures are still calculated, but in less than $10 \%$ of the computational time. This provides a useful method of obtaining an efficient simulation if internal stress information is not required.

\section{Conclusions and Future Work}

A dynamic FE model of a TKR was presented that incorporates the femoral, tibial and patellar components. The model simulated a dynamic extension motion under quadriceps control. A validation experiment was conducted and the model was able to accurately predict the kinematics of both the tibiofemoral and patellofemoral joints along with predicting the net forces and moments applied to the femur. To the best of our knowledge, this is the first experimental validation of a dynamic FE model of a TKR to employ simultaneous kinematic and force data. 
Such models are valuable because they can be used to predict physiological motion prior to surgery. This is of particular importance for patients with significant deformities or poor overall health, both of which are predictors for poor surgical outcomes. A dynamic knee model is also of great use in the design of new prostheses, as it makes it possible to reduce or eliminate expensive, time-consuming physical tests.

Future work will include the addition of physiologically realistic ligament structures and bone/ligament interaction. Further experimental validation will be conducted in vitro, including simulation of TKR surgeries and predicting outcomes of simulated muscle-controlled loading from MRI-derived ligament data.

\section{References}

1. Stulberg, S.D., Loan, P., Sarin, V.: Computer-assisted navigation in total knee replacement: Results of an initial experience in thirty-five patients. Journal of Bone and Joint Surgery 84 (2002) 90-98

2. Victor, J., Hoste, D.: Image-based computer-assisted total knee arthroplatsy leads to lower variability in coronal alignment. Clinical Orthopaedics and Related Research 428 (2004) 131-139

3. Giddings, V.L., Kurtz, S.M., Edidin, A.A.: Total knee replacement polyethylene stresses during loading in a knee simulator. Journal of Tribology-Transactions of the ASME 123 (2001) 842-847

4. Otto, J.K., Callaghan, J.J., Brown, T.D.: The coventry award paper - Mobility and contact mechanics of a rotating platform total knee replacement. Clinical Orthopaedics and Related Research 392 (2001) 24-37

5. Godest, A.C., Beaugonin, M., Haug, E., Taylor, M., Gregson, P.J.: Simulation of a knee joint replacement during a gait cycle using explicit finite element analysis. Journal of Biomechanics 35 (2002) 267-275

6. Taylor, M., Barrett, D.S.: Explicit finite element simulation of eccentric loading in total knee replacement. Clinical Orthopaedics and Related Research 414 (2003) 162-171

7. Halloran, J.P., Petrella, A.J., Rullkoetter, P.J.: Explicit finite element modeling of total knee replacement mechanics. Journal of Biomechanics 38 (2005) 323-331

8. Wilson, D.R., Apreleva, M.V., Eichler, M.J., Harrold, F.R.: Accuracy and repeatability of a pressure measurement system in the patellofemoral joint. Journal of Biomechanics 36 (2003) 1909-1915 